\title{
Journal of Sustainable Development of Energy, Water and Environment Systems - Volume III
}

\author{
Neven Duić \\ Editor-in-Chief, Journal of Sustainable Development of Energy, Water and \\ Environment Systems
}

The Journal of Sustainable Development of Energy, Water and Environment Systems - JSDEWES is an international journal dedicated to the improvement and dissemination of knowledge on methods, policies and technologies for increasing the sustainability of development by de-coupling growth from natural resources and replacing them with knowledge based economy, taking into account its economic, environmental and social pillars, as well as methods for assessing and measuring sustainability of development, regarding energy, transport, water, environment and food production systems and their many combinations.

In total 32 manuscripts were published in Volume III, all of them reviewed by at least two reviewers. The Journal of Sustainable Development of Energy, Water and Environment Systems would like to thank reviewers for their contribution to the quality of the published manuscripts.

1. Prof. Paola Acevedo Pabón

2. Dr. Youssef Ahmad Youssef

3. Prof. Skender Ahmetaj

4. Prof. Elena Andreevska

5. Dr. Aleksandra Anić Vučinić

6. Dr. Esin Apaydin Varol

7. Prof. Jurij Avsec

8. Prof. Brian Azzopardi

9. Prof. Milun Babić

10. Dr. Ipek Barut

11. Prof. Simona Colajanni

12. Dr. Lidija Čuček

13. Mr. Boris Ćosić

14. Prof. Gradimir Danon

15. Mr. Aleksandar Dedinec

16. Prof. Slaven Dobrović

17. Prof. Mariusz Dudziak

18. Dr. Mansour Emtir

19. Mr. Bredo Erichsen

20. Prof. Germán Ferreira

21. Prof. Mihajlo Firak

22. Dr. Muriel Gavira

23. Prof. Giuseppe Genon

24. Prof. Anita Grozdanov

25. Prof. Dušan Gvozdenac

26. Dr. Eduard Hanslík

27. Prof. Pavel Hasal

28. Dr. Takashi Homma

29. Dr. Andrea Hublin

30. Prof. Aleksandar Jovović

31. Dr. Skender Kabashi

32. Prof. Yuya Kajikawa

33. Prof. Emmanuel Kakaras

34. Dr. Şiir Kilkiş

35. Ms. Aleksandra Krkoleva
36. Prof. Zainuddin Manan

37. Dr. Kenichi Matsumoto

38. Ms. Barbara Mendecka

39. Mr. Hrvoje Mikulčić

40. Prof. Alberto Mirandola

41. Dr. Karel Mulder

42. Prof. Michael Narodoslawsky

43. Prof. Matilde Pietrafesa

44. Dr. Fernando Prado Jr.

45. Prof. Nikola Rajaković

46. Prof. Predrag Rašković

47. Prof. Md Mastufizur Rhaman

48. Prof. Nabil Rofail

49. Dr. Jaqueline Saavedra Rueda

50. Dr. Dana Sitanyiova

51. Prof. Izet Smajević

52. Prof. Ingo Stadler

53. Prof. Svetlana Stevović

54. Prof. Rodney Stewart

55. Prof. Alexandre Szklo

56. Dr. Ricardo Teixeira

57. Dr. Agnieszka Terelak-Tymczyna

58. Prof. Željko Tomšić

59. Dr. Mauro Villarini

60. Dr. Katarzyna Zwarycz-Makles 